\title{
Design of Efficient Algorithms for Video Surveillance Applications using Artificial Intelligence
}

\section{Mohana}

Research Scholar, Electronics and Communication Engg Assistant Professor, Electronics and Telecommunication Engg RV College of Engineering, Bengaluru-560059, India mohana@rvce.edu.in

Abstract - Object detection and tracking algorithms such as YOLO(You Look Only Once Version V1 to V3), SSD and SORT implemented on COCO and indigenous data set for traffic surveillance and evaluated using the performance metrics such as True Positive (TP), True Negative (TN), False Positive (FP), False Negative (FN), mean Average Precession (mAP). The designed $\mathrm{CNN}$ trained on dataset (small and large) had similar performance on test dataset, however the CNN trained on the large datasets that had larger intra-class variations was able classify a greater number of vehicles belonging to light and two-wheeler class. It achieved a validation accuracy of $98 \%$. VGG16 achieved an accuracy of 97\% followed by MobileNetV2 and InceptionV3 with $75 \%$ and $50 \%$ accuracy respectively.

Keywords- YOLOv3, Single Shot Detector (SSD), Convolution Neural Network (CNN), Artificial intelligence, VGG16, InceptionV3, MobileNetV2, FPGA Zynq XC7Z020, DSP Embest Dev Kit 8500D.

\section{INTRODUCTION}

Video has become one of the most important media for information exchange due to its availability and accessibility. It is extensively used in many applications, of which video surveillance has exponential usage in time that is accelerated by the advent of large storage capacity and high-performance computers capable of performing computer vision tasks within no time. Video surveillance has become a necessity and hardly are there any fields that is untouched by it. The rich resource is now accelerating the researchers to excavate more details form the images. These developments are due to the state-of the-art methods like Convolutional Neural Network (CNN).Traffic Surveillance has become one of the most important applications of video surveillance. Automatic Traffic signals can minimize the chaos in a densely populated country like India. The necessity for such automation is to detect, track and classify vehicles on Indian roads. Deep Learning echniques can be used to train object classifiers that can be used in real time to detect, track and classify the objects. There are many algorithms used in literature. As per the exhaustive literature survey of over $200+$ papers, in this research work object detection is implemented using YOLOV3 and SSD algorithm. Objects are tracked using SORT (simple online real time tracking) method. Indian Urban Vehicles dataset used to train single object classifiers that can classify the images of vehicles into four classes namely auto, heavy, light and twowheeler. Convolutional Neural Network $(\mathrm{CNN})$ was designed and trained on the two subsets being small and large training datasets in order to evaluate the performance of CNN on more

\author{
H. V. Ravish Aradhya \\ Research Advisor, Professor \\ Electronics and Communication Engineering \\ RV College of Engineering, Bengaluru-560059, India \\ ravisharadhya@rvce.edu.in
}

and less amount of data. Transfer Learning using Visual Geometry Group16 (VGG16), InceptionV3 and MobileNetV2 used on small training dataset to evaluate the performance of these state-of-the-networks against the designed CNN trained from scratch. The performance of these models are evaluated using metrics such as accuracy, loss, precision, recall and f1score. Confusion matrix is used to evaluate the model on a test dataset.

\section{MOTIVATION}

Surveillance is the emerging concept in current technology, as it plays a vital role in monitoring keen activities at the nooks and corner of the world. Lack of intelligence in existing system has driven enormous amount of research in myriad areas of video surveillance to make the systems more intelligent, robust and autonomous. With the large availability of video/image data from past one decade, Artificial Intelligence has the most promising results. The applications include and is not limited to smart city, public safety, traffic related applications, restricted area and border security and some general indoor and outdoor applications. There is a need for designing efficient detection, tracking and classification algorithm that carefully addresses the trade-off between speed, accuracy and size by fine-tuning the performance parameters of the learning algorithms to make it suitable for video surveillance (traffic) applications.

\section{DESIGN AND IMPLEMENTATION}

A. Object detection and Tracking

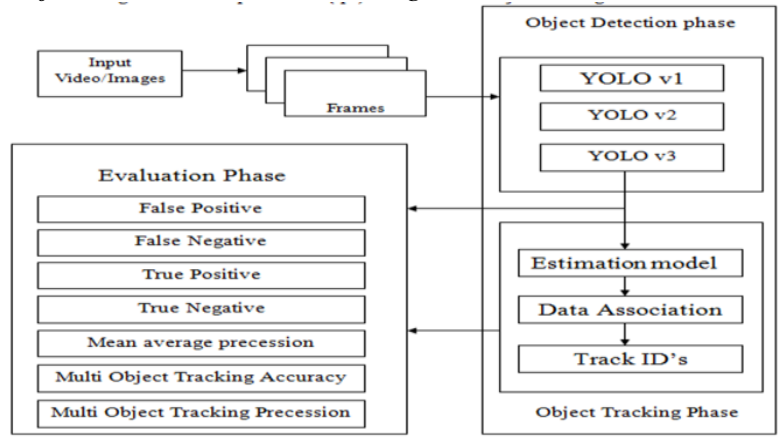

Fig.1.Block diagram of object detection and object tracking.

The video sequence is divided into frames as shown in figure 1. The frames are given as input to YOLOv3 (the best version of YOLO is considered for detection). The Bounding box output of YOLOv3 as input to the Object tracking phase (SORT is used). Data association is the process that makes the SORT algorithm best suitable for object tracking. Track Identities is assigned to the detected bounding boxes, trajectory of which needs to be found. The bounding box from the object detection phase is used as reference to analyze the 
performance metric. Metrics such as false positive, false negative, true positive, true negative, mean average precession, MOTA (Multi Object Tracking Accuracy) and MOTP (Multi Object Tracking Precession) is analyzed to appreciate the accuracy of the detector and tracker. An appropriate object classifier for classification of Indian urban vehicles is designed and the performance is analysed with other state-of-the-art neural networks. For single object a $\mathrm{CNN}$ is trained from scratch on Large and Small Datasets and transfer learning is used to train VGG16, InceptionV3 and MobileNetV2 on small datasets. Finally, SSD is trained on labelled dataset for classification.

\section{B. Dataset}

The specifications of Urban Vehicle Dataset [1] is tabulated in table II and the number of images considered for each class is tabulated in Table III. In total there are 64339 images belonging to 4 classes that are taken under different times of the day and capturing conditions, including NIR images. The images are classified based on the utility and size of vehicles. The auto class has images of 3 wheelers; Heavy class includes buses, trucks; Light class has cars, SUVs and sedans and two wheelers include motorcycles and bicycles. Most of the images has only one object belonging to its respective class. In this research work RGB_Day images are considered and divided into two subset of classes small and large for training and testing.

TABLE I DATASET SPECIFICATIONS

\begin{tabular}{|c|c|c|c|c|c|}
\hline \multicolumn{3}{|c|}{ Source } & \multicolumn{3}{|c|}{ Traffic Monitoring Cameras } \\
\hline \multicolumn{3}{|c|}{ Image Type } & \multicolumn{3}{|c|}{ RGB, NIR } \\
\hline \multicolumn{3}{|c|}{ Image Extension } & \multicolumn{3}{|c|}{ Jpg } \\
\hline \multicolumn{3}{|c|}{ Image Dimension } & \multicolumn{3}{|c|}{ Variable Size } \\
\hline \multicolumn{3}{|c|}{ Image Quality } & \multicolumn{3}{|c|}{ Medium to Blurred } \\
\hline \multicolumn{6}{|c|}{ TABLE II URBAN VeHICLE DATASET } \\
\hline Directory & Autos & Heavy & Light & Two Wheelers & Total \\
\hline RGB Day & 2530 & 2915 & 12927 & 13340 & 31712 \\
\hline RGB Evening & 3122 & 3709 & 14654 & 10027 & 31512 \\
\hline NIR & 124 & 127 & 524 & 340 & 1115 \\
\hline \multicolumn{6}{|c|}{ TABle III TeSt-Train SPlit fOR Vehicle Dataset SMALl } \\
\hline RGB_Day & Autos & Heavy & Light & Two wheelers & Total \\
\hline Training Set & 2000 & 2400 & 5600 & 5000 & 15000 \\
\hline Test Set & 500 & 500 & 500 & 500 & 2000 \\
\hline
\end{tabular}

TABle IV TESt-Train SPlit FOR Vehicle DATASET LARGE

\begin{tabular}{|c|c|c|c|c|c|}
\hline RGB Day & Autos & Heavy & Light & Two wheelers & Total \\
\hline Training Set & 2030 & 2415 & 12427 & 12840 & 29712 \\
\hline Test Set & 500 & 500 & 500 & 500 & 2000 \\
\hline
\end{tabular}

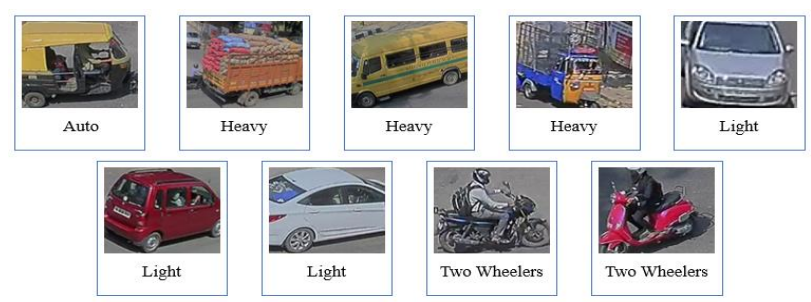

Fig.2.Sample Images of the Dataset [1].

single object classifier: Designed CNN has 2 Convolutional layers to extract the features, 2 Max Pooling Layers to reduce the image dimensionality, flatten layer converts the output into column tensor and 2 dense layers are created to differentiate the objects identified. When the image has multiple objects, localization along with classification constitutes the task of object detection; state- of- the -art neural networks like darknet will perform end-to-end operation in detecting the objects.

TABLE V HYPER PARAMETERS OF CNN

\begin{tabular}{|c|c|}
\hline Hyper Parameters & Values \\
\hline Input Size & $128 * 128 * 3$ \\
\hline Filter Size & $32(3 * 3)$ \\
\hline Activation & ReLU and softmax \\
\hline Optimizer & SGD \\
\hline Learning Rate & 0.001 \\
\hline Batch Size & 32 \\
\hline Epoch & 10 \\
\hline Layers & 7 \\
\hline
\end{tabular}

TABLE VI CONVOLUTIONAL NEURAL NETWORK DESIGN

\begin{tabular}{|c|c|c|}
\hline Layer & Output shape & Parameters \\
\hline Convolution & $126 * 126 * 32$ & 896 \\
\hline Max pooling & $63 * 63 * 32$ & 0 \\
\hline Convolution & $61 * 61 * 32$ & 9248 \\
\hline Max Pooling & $30 * 30 * 32$ & 0 \\
\hline Flatten & 288000 & 0 \\
\hline Dense & 128 & 3686528 \\
\hline Dense & 4 & 516 \\
\hline
\end{tabular}

\section{Performance metrics}

The choice of performance metrics will influence the analysis of the algorithms. This helps in identifying the reasons for mis-classifications so that it can be corrected by taking necessary measures.

Confusion Matrix

$$
\begin{aligned}
& \begin{array}{|c|c|c|}
\hline & \text { Classl Predicted } & \text { Class 2 Predicted } \\
\hline \text { Classl Actual } & \begin{array}{c}
\text { True Positive (TP) } \\
\text { Correct Decision }
\end{array} & \begin{array}{c}
\text { False Negative (FN) } \\
\text { Type 1 error }
\end{array} \\
\hline \text { Class 2 Actual } & \begin{array}{c}
\text { False Positive (FP) } \\
\text { Type 2 error }
\end{array} & \begin{array}{c}
\text { True Negative (TN) } \\
\text { Correct Decision }
\end{array} \\
\hline
\end{array} \\
& \text { Precision }=\frac{T P}{T P+F P} \\
& \text { Recall }=\frac{T P}{T P+F N} \\
& F-1 \text { Score }=2 * \frac{\text { Preciison } * \text { Recall }}{\text { Precision }+R e c a l l} \\
& m A P=\frac{1}{\text { No.of divisions }} \sum_{r €(1,0.1,0.001)} p_{\text {interp }}(r)
\end{aligned}
$$

Object detection and Classifier: CNN Design

TABLE VII CNN HYPER PARAMETER DETAILS

\begin{tabular}{|c|c|}
\hline Hyperparameter & Type/Value \\
\hline Image Size & $64 \times 64$ \\
\hline Image Channels & 3 (RGB) \\
\hline Kernel Initializer & Uniform Distribution \\
\hline Number of Kernels & 32 \\
\hline Kernel Size & $3 \times 3$ \\
\hline Activation Functions & Relu, SoftMax \\
\hline Optimizer & Adam \\
\hline Batch Size & 32 \\
\hline Epochs & 25 \\
\hline Learning Rate & 0.001 \\
\hline Neurons in Hidden Layer & $\mathbf{1 2 8}$ \\
\hline
\end{tabular}

TABLE VIII CNN ARCHITECTURE

\begin{tabular}{|c|c|c|c|}
\hline Layer & Operation & Output Shape & Trainable Parameters \\
\hline 1 & Convolution & $62 \times 62 \times 32$ & 896 \\
\hline 2 & Max Pooling & $31 \times 31 \times 32$ & 0 \\
\hline 3 & Convolution & $29 \times 29 \times 32$ & 9,248 \\
\hline 4 & Max Pooling & $14 \times 14 \times 32$ & 0 \\
\hline 5 & Flatten & 6272 & 0 \\
\hline 6 & Dense & 128 & $8,02,944$ \\
\hline 7 & Dense & 4 & 516 \\
\hline
\end{tabular}

Transfer Learning

TABLE IX NETWORK DESIGN-TRANSFER LEARNING

\begin{tabular}{|c|c|c|c|c|c|}
\hline Network & $\begin{array}{c}\text { Frozen } \\
\text { layers }\end{array}$ & Input Size & $\begin{array}{c}\text { Total } \\
\text { Parameters }\end{array}$ & $\begin{array}{c}\text { Non-Trainable } \\
\text { Parameters }\end{array}$ & $\begin{array}{c}\text { Trainable } \\
\text { Parameters }\end{array}$ \\
\hline VGG 16 & 18 & $128 \times 128 \times 3$ & $1,57,63,908$ & $1,47,14,688$ & $10,49,220$ \\
\hline Inception V3 & 310 & $75 \times 75 \times 3$ & $2,20,65,572$ & $2,18,02,784$ & $2,62,788$ \\
\hline MobileNet V2 & 154 & $96 \times 96 \times 3$ & $24,22,468$ & $22,57,984$ & $1,64,484$ \\
\hline
\end{tabular}

TABLE X HYPER PARAMETER DETAILS FOR TRANSFER LEARNING

\begin{tabular}{|c|c|}
\hline Hyperparameter & Type/Value \\
\hline Image Channels & 3 (RGB) \\
\hline Kernel Initializer & Uniform Distribution \\
\hline Optimizer & Adam \\
\hline Batch Size & 32 \\
\hline Epochs & 10 \\
\hline Learning Rate & 0.001 \\
\hline Neurons in Hidden Layer & 128 \\
\hline
\end{tabular}

SSD: Transfer learning is employed for training SSD to perform object detection. The SSD model trained on COCO dataset is used for this purpose. The training of object detectors requires labelled datasets that has information regarding the location of the object in an image along with its class. 


\begin{tabular}{|c|c|c|c|c|c|}
\hline RGB_Day & Autos & Heavy & Light & Two wheelers & Total \\
\hline Training Set & 2000 & 2000 & 4000 & 3000 & 11000 \\
\hline Test Set & 500 & 500 & 500 & 500 & 2000 \\
\hline
\end{tabular}

\begin{tabular}{|c|c|} 
TABLE XII HYPER PARAMETER DETAILS FOR SSD \\
\hline Hyperparameter & Type/Value \\
\hline Image Size & $300 \times 300$ \\
\hline Image Channels & 3 (RGB) \\
\hline Kernel Initializer & Random Normal Initializer \\
\hline Kernel size & $3 \times 3$ \\
\hline Optimizer & Adam \\
\hline Batch Size & 32 \\
\hline Learning Rate & 0.0002 \\
\hline L2 Regularizer & 0.00004 \\
\hline
\end{tabular}

IV. SIMULATION RESULTS AND ANALYSIS

A. Results of object detection and Tracking

Single object classification is performed on the state-of -theart network called CNN. The network designed consists of 3,697,188 tunable parameters.

TABLE XIII SINGLE OBJECT CLASSIFIER SUMMARY

\begin{tabular}{|c|c|}
\hline Attribute & Values \\
\hline Total training Time & 110 minutes \\
\hline Train Accuracy & 0.9821 \\
\hline Validation Accuracy & 0.9941 \\
\hline Train Loss & 0.0591 \\
\hline Validation Loss & 0.0219 \\
\hline
\end{tabular}
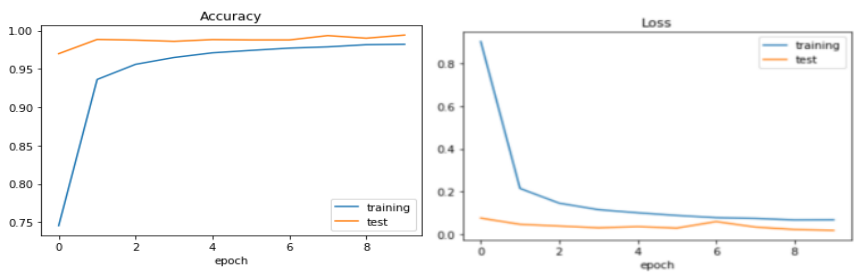

Fig.3. (a) Accuracy plot of CNN (b) Loss plot for CNN

The Accuracy of the network is gradually increasing, and loss curve is gradually decreasing with increase in number of epochs.

\section{Confusion matrix}

The performance of the classification model is measured using confusion matrix. The classifier is trained for four classes such as auto, heavy, light, two-wheeler.

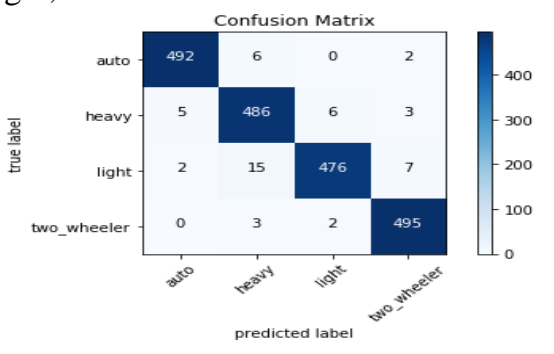

Fig.4.confusion matrix for $\mathrm{CNN}$

Out of 500 images from each class 492 autos, 486heavy vehicles, 476 light vehicles and495 two-wheelers have been correctly detected.

TABLE XIV PERFORMANCE METRICS OF CNN
\begin{tabular}{|c|c|c|}
\hline Class & Precession & Recall \\
\hline Auto & 0.99 & 0.98 \\
\hline Heavy & 0.95 & 0.97 \\
\hline Light & 0.98 & 0.95 \\
\hline Two-wheeler & 0.98 & 0.99 \\
\hline
\end{tabular}

\section{Results of YOLOv3}

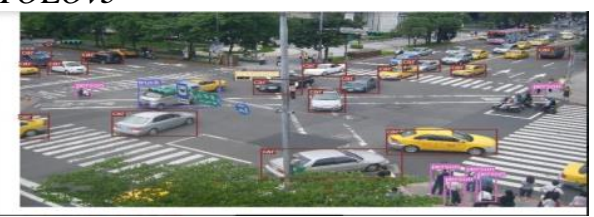

Fig.5. YOLOv3 results
It can detect objects of any size and images captured from various camera angle and distance. This attribute is because of the FPN used in YOLOv3.
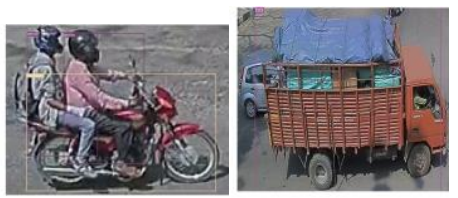

Fig.6. RGB_Day image detections
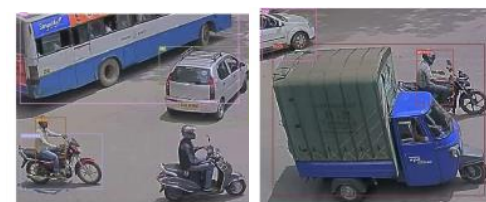

Fig.7. RGB_Evening Images detections
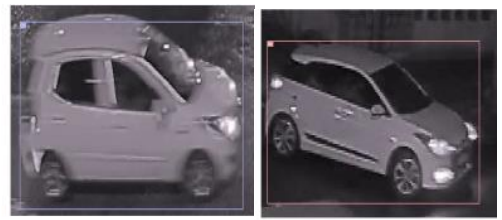

Fig.8.NIR Images detections

The dataset consists of three different kinds of images such as day images, evening images and (Near Infrared) NIR images are considered.

SORT Analysis

It is implemented for 30 FPS with.mp4 video formatting. The IoU thresholding is considered as 0.3 . Reduced ID switches Increase in Mostly tracked ground truths and decrease in mostly lost ground truths is achieved by associating appearance information.

Performance metrics: For object car

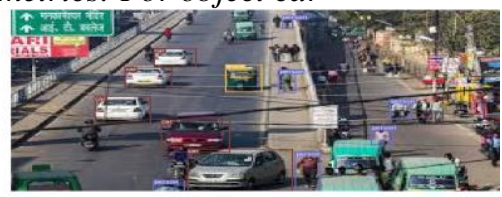

Fig. 9.Car Detection

The precession result is high since the objects to be detected are not occluding one another and the recall is 0.8333 since the FN value is 1 as the auto in the image is detected but identified as truck. As the result of misclassification, the recall value suffers the loss. Since the precision is high the mAP value is $100 \%$ for the given class and considered image.

TABLE XV PERFORMANCE ANALYSIS OF OBJECT DETECTION (CARS)

\begin{tabular}{|c|c||c|c|c|c|c|}
\hline & TP & TN & FP & FN & Precision & Recall \\
\hline IoU at. 25 & 5 & 0 & 0 & 1 & 1 & 0.8333 \\
\hline IoU at .50 & 5 & 0 & 0 & 1 & 1 & 0.8333 \\
\hline IoU at. 75 & 4 & 0 & 0 & 1 & 1 & 0.8333 \\
\hline
\end{tabular}

B. Results of object detection and Classifier

CNN Results and Analysis

TABLE XVI CNN OUTPUT DETAILS

\begin{tabular}{|c|c|c|}
\hline Measure & Value for Small Dataset & Value for Large Dataset \\
\hline Time forsingleepoch & 81 seconds & 300 seconds \\
\hline Training Accuracy & 0.9896 & 0.9936 \\
\hline Validation Accuracy & 0.9853 & 0.9812 \\
\hline Training Loss & 0.0295 & 0.0193 \\
\hline Validation Loss & 0.04803 & 0.06175 \\
\hline
\end{tabular}

TABLE XVII CNN CLASSIFICATION REPORT

\begin{tabular}{|c||c|c|c|c|c|c|}
\hline \multicolumn{1}{|c|}{} & \multicolumn{3}{c|}{ Small Dataset } & \multicolumn{3}{c|}{ Large Dataset } \\
\cline { 2 - 7 } & precision & recall & fl-score & precision & recall & fl-score \\
\hline auto & 0.99 & 0.99 & 0.99 & 0.99 & 0.99 & 0.99 \\
\hline heavy & 0.97 & 0.95 & 0.96 & 0.99 & 0.93 & 0.96 \\
\hline light & 0.96 & 0.98 & 0.97 & 0.95 & 0.99 & 0.97 \\
\hline two_wheeler & 0.99 & 0.99 & 0.99 & 0.98 & 1.00 & 0.99 \\
\hline
\end{tabular}

Better accuracy and loss values achieved on large datasets and the model is more generalized when trained on Large Dataset. The precision and recall scores are comparable in both cases. 

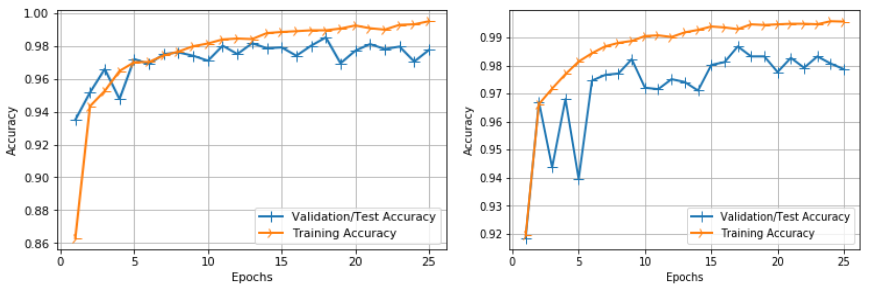

Fig.10.CNN Accuracy Plots for Small (Left) and Large (Right) Datasets.
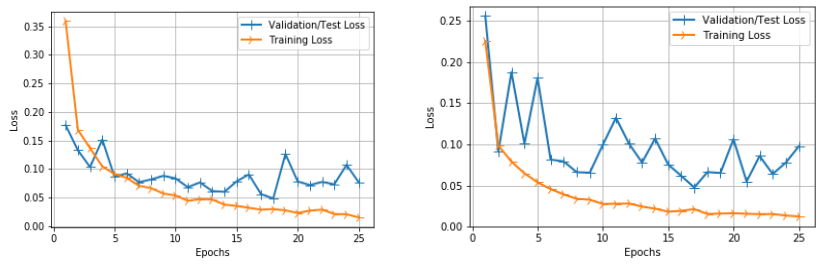

Fig.11. CNN Loss Plots for Small (Left) and Large (Right) Datasets
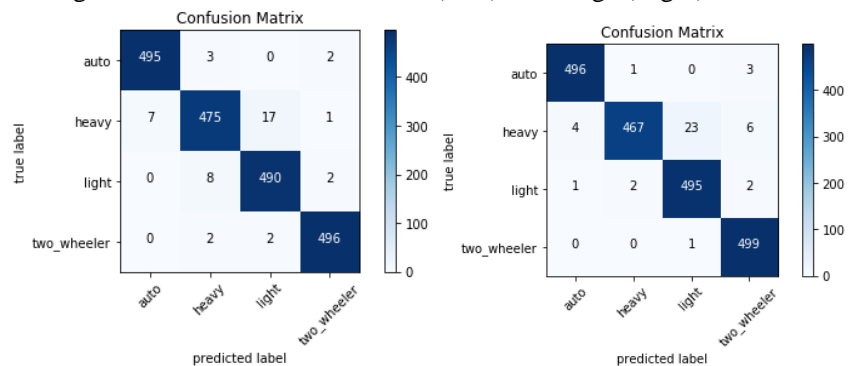

Fig.12. CNN Confusion Matrix for Small (Left) and Large (Right) Datasets
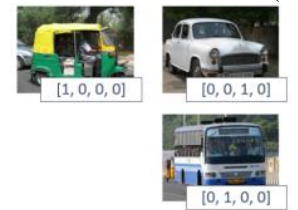

Fig.13. Single Prediction by CNN

A total of 2000 images distributed equally among four classes i.e. 500 images per class is considered for prediction using CNN model. It is evident that both the models have approximately similar performance. The respective positions of the class will be high in the output; here all the classes are predicted accurately.

TABLE XVIII TRANSFER LEARNING OUTPUT DETAILS
\begin{tabular}{|c|c|c|c|}
\hline Measure & VGGl6 & InceptionV3 & MobileNetV2 \\
\hline Time for single epoch & 3000 seconds & 250 seconds & 200 seconds \\
\hline Training Accuracy & 0.9780 & 0.7753 & 0.9757 \\
\hline Validation Accuracy & 0.9853 & 0.5086 & 0.7693 \\
\hline Training Loss & 0.0662 & 0.5930 & 0.0704 \\
\hline Validation Loss & 0.0453 & 2.1965 & 0.8965 \\
\hline
\end{tabular}

TABLE XIX TRANSFER LEARNING CLASSIFICATION REPORT

\begin{tabular}{|c|c|c|c|c|c|c|c|c|c|}
\hline & \multicolumn{3}{|c|}{ VGGl6 } & \multicolumn{3}{c|}{ InceptionV3 } & \multicolumn{3}{c|}{ MobileNetV2 } \\
\cline { 2 - 10 } & precision & recall & $\begin{array}{c}\text { fl- } \\
\text { score }\end{array}$ & precision & recall & $\begin{array}{c}\text { fl- } \\
\text { score }\end{array}$ & precision & recall & $\begin{array}{c}\text { fl- } \\
\text { score }\end{array}$ \\
\hline auto & 1.00 & 0.95 & 0.98 & 0.81 & 0.33 & 0.47 & 0.99 & 0.30 & 0.46 \\
\hline heavy & 0.96 & 0.97 & 0.96 & 0.61 & 0.13 & 0.22 & 0.96 & 0.55 & 0.70 \\
\hline light & 0.97 & 0.99 & 0.98 & 0.36 & 0.95 & 0.52 & 0.46 & 1.00 & 0.63 \\
\hline two_wheeler & 0.99 & 1.00 & 1.00 & 0.56 & 0.42 & 0.48 & 0.99 & 0.97 & 0.98 \\
\hline
\end{tabular}

The f1-score is good for VGG16 followed by MobileNetV2 and InceptionV3 which has poor performance on heavy and auto class and performs fairly on light and two_wheeler class. The MobielNetV2 performs best on two_wheeler class but fails on the rest of the class.
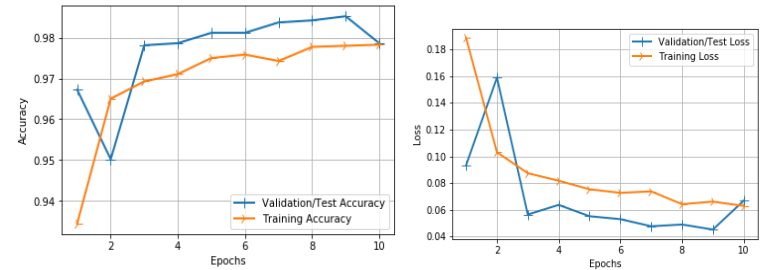

Fig.14. VGG16 Accuracy (Left) and Loss (Right) Plots
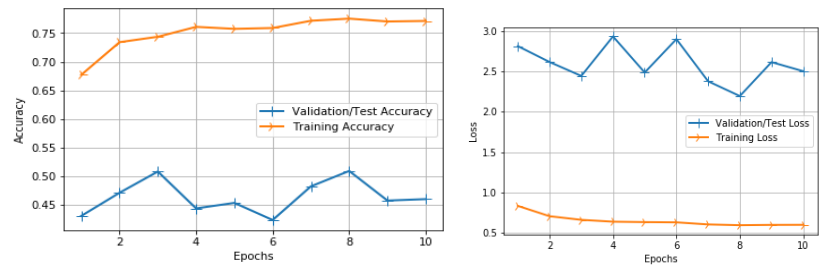

Fig.15. InceptionV3 Accuracy (Left) and Loss (Right) Plots
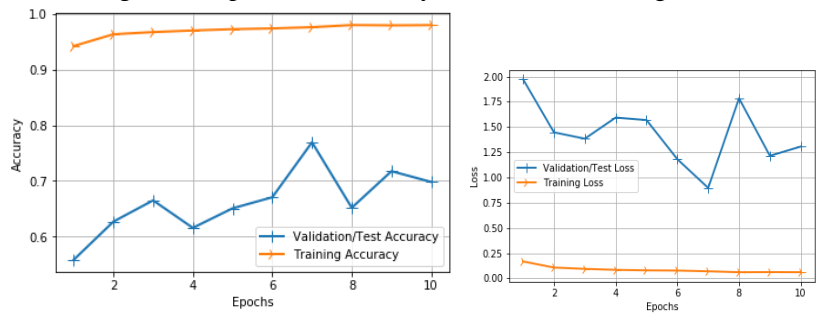

Fig.16. MobileNetV2 Accuracy (Left) and Loss (Right) Plots Better accuracy and loss values is achieved on VGG16 model. It can be inferred that the validation loss values for VGG16 will converge better when trained for a greater number of epochs.
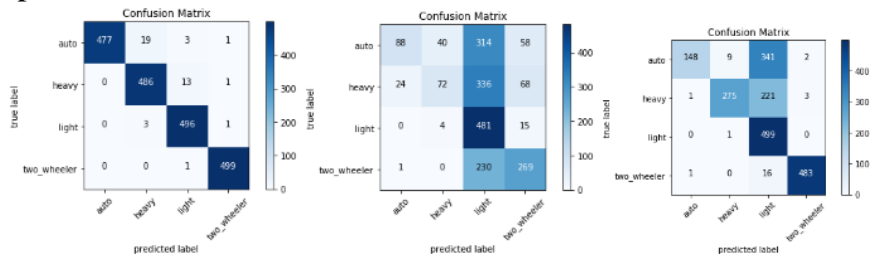

Fig.17. Confusion Matrix for VGG16, InceptionV3 and MobileNetV2

\section{CONCLUSIONS}

The selection of suitable algorithm for a particular task is always a trade-off between speed and accuracy.The single class classifier trained on the Indigenous dataset achieved a validation accuracy of $99.41 \%$. VGG16 has better performance followed by MobileNetV2 and InceptionV3. MobileNet can be tuned properly to achieve descent levels of accuracy since it trains faster than VGG16 and can be deployed on Mobile Platforms. The SSD implementation for object detection is a good choice since SSD uses VGG16 as a classifier. The CNN model trained on large and small datasets for single object classification, achieved a validation accuracy of $98 \%$. The VGG16 model trained using transfer learning on small datasets reached a validation accuracy of $97 \%$ followed by MobileNetV2 with $76 \%$ and InceptionV3 with $50 \%$ validation accuracy. Detection and tracking algorithms are implemented on DSP board Embest Dev kit 8500D and FPGA Zynq XC7Z020.

\section{REFERENCES}

[1] H. S. Bharadwaj, S. Biswas and K. R. Ramakrishnan. "A large scale dataset for classification of vehicles in urban traffic scenes", Proceedings of the Tenth Indian Conference on Computer Vision, Graphics and Image Processing,ACM,2016.

[2] Balamurugan S.A, Rajagopal B.G, and Parasuram K, "A robust framework to detect moving vehicles in different road conditions in India", Journal of Theoretical and Applied Information Technology, vol. 96, no. 1, pp. 1-14, 2019.

[3] P. Wang, L. Li, Y. Jin and G. Wang, "Detection of unwanted traffic congestion based on existing surveillance system using in freeway via a CNN-architecture trafficnet," 13th IEEE Conference on Industrial Electronics and Applications (ICIEA), Wuhan, 2018, pp. 1134-1139.

[4] Mohana, H. V. Ravish Aradhya, "Object Detection and Classification Algorithms using Deep Learning for Video Surveillance Applications" International Journal of Innovative Technology and Exploring Engineering (IJITEE), ISSN: 2278-3075, Volume-8 Issue-8 June, pp.386-395, 2019.

[5] Mohana, H. V. Ravish Aradhya,"Real-Time Implementation of object detection and Tracking on FPGA for video surveillance applications" 2016 IEEE International Conference on Distributed Computing,VLSI, Electrical Circuits and Robotics(DISCOVER) National Institute of Technology surathkal, Karnataka, pp. 289-295, 2016. 